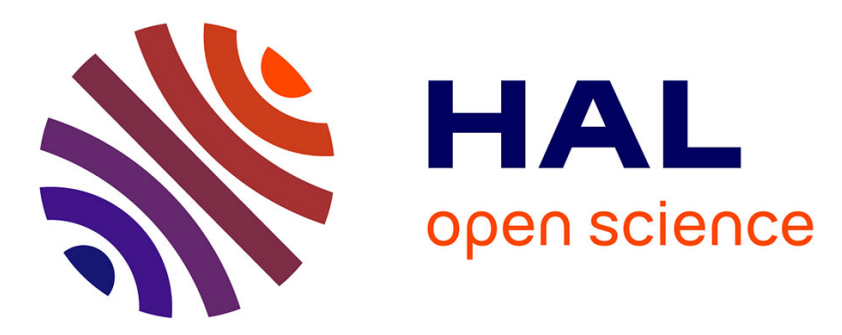

\title{
Real-time layer-based computer-generated hologram calculation for the Fourier transform optical system
}

\author{
Antonin Gilles, Patrick Gioia
}

\section{To cite this version:}

Antonin Gilles, Patrick Gioia. Real-time layer-based computer-generated hologram calculation for the Fourier transform optical system. Applied optics, 2018, 57 (29), pp.8508-8517. 10.1364/AO.57.008508 . hal-03361718

\section{HAL Id: hal-03361718 https://hal.science/hal-03361718}

Submitted on 1 Oct 2021

HAL is a multi-disciplinary open access archive for the deposit and dissemination of scientific research documents, whether they are published or not. The documents may come from teaching and research institutions in France or abroad, or from public or private research centers.
L'archive ouverte pluridisciplinaire HAL, est destinée au dépôt et à la diffusion de documents scientifiques de niveau recherche, publiés ou non, émanant des établissements d'enseignement et de recherche français ou étrangers, des laboratoires publics ou privés. 


\title{
Real-time layer-based Computer-Generated Hologram calculation for the Fourier Transform Optical System
}

\author{
Antonin Gilles $^{1} \quad$ Patrick Gioia ${ }^{1,2}$ \\ ${ }^{1}$ IRT $\mathrm{b}<>$ com \\ Cesson-Sévigné \\ 2 Orange Labs \\ Rennes \\ France \\ France
}

\begin{abstract}
With the growing interest for Augmented Reality devices, holography is often considered as a promising technology to overcome the focus issues of conventional stereoscopic displays. To enlarge the field-of-view of holographic HeadMounted Displays, a Fourier Transform Optical System (FTOS) has been proposed. However, since the scene geometry is distorted by the FTOS, it is necessary to compensate the position of each scene point during the hologram computation, resulting in long calculation times. In this paper, we propose a real-time Computer-Generated Hologram (CGH) calculation method for the FTOS. Whereas previously proposed methods used a ray-tracing approach to compensate the distortion induced by the FTOS, our proposed method relies on a layer-based approach. Experimental results show that our method is able to compute holograms of resolution $(3840 \times 2160)$ in real-time at 24 frames per second, enabling its use in Augmented Reality applications.
\end{abstract}

Keywords: Computer-Generated Hologram, Color holography, Three-dimensional imaging

\section{Introduction}

With the recent advances in Head-Mounted Display (HMD) devices, Augmented Reality (AR) has attracted a significant attention during the last decade [1]. Thanks to its attractive features in terms of visualization, AR has found application in many fields, including medicine [2], architecture [3], navigation [4] and defense [5].

However, conventional AR glasses still suffer from several technical challenges that prevent them from being widely used by the general public. One of their most important limitation is their inability to provide a natural and realistic depth illusion to the viewer. Indeed, to create the threedimensional (3D) illusion, conventional AR headsets usually

2016 Optical Society of America. One print or electronic copy may be made for personal use only. Systematic reproduction and distribution, duplication of any material in this paper for a fee or for commercial purposes, or modifications of the content of this paper are prohibited. http://dx.doi.org/10.1364/AO.55.005459 rely on the stereoscopic technique, which cannot provide the accommodation depth cue. When using such glasses, the viewer has to focus on a fixed focal plane to perceive sharp virtual objects. As a consequence, most of the time it is impossible to focus on real and virtual objects simultaneously, which significantly complicates natural interaction with virtual content. Moreover, it creates a mismatch between the vergence and accommodation cues, known as the vergenceaccommodation conflict, which leads to visual discomfort, eyestrain and headaches [6].

To overcome these limitations, several accommodationsupporting display technologies have been proposed in recent years, including varifocal [7], multifocal [8], focal surfaces [9], light field [10] and holographic displays [11]. Among these new technologies, holography is often considered as one of the most promising, since it provides the most natural and realistic depth illusion to the human eye [12]. In $[13,14]$, the authors successfully designed compact see-through holographic headsets able to provide the accommodation depth cue. However, since the maximum viewing angle of a hologram is limited by its pixel pitch, these HMDs were unable to provide a field of view (FOV) wider than a few degrees.

To enlarge the FOV, a Fourier Transform Optical System (FTOS), in which a converging lens is set in front of the hologram, has been proposed [15]. This system is equivalent to a conventional optical system illuminated by a converging spherical wave instead of a plane wave [16]. In contrast with other FOV expansion solutions, such as using multiple displays [17], eye-tracked viewing windows [18] or temporal multiplexing [19], the FTOS and spherically illuminated holographic displays present the advantage of being simple and lightweight optical systems which can easily fit into AR glasses [20]. However, since the scene geometry is distorted either by the lens or the converging wave, it is necessary to compensate the position of each scene point during the Computer-Generated Hologram (CGH) calculation [21], and real-time computation is still very challenging.

In [22], the authors proposed to accelerate the CGH calculation by taking into account temporal redundancies during viewpoint parallel shift and rotation. However, while being more than twice as fast as the algorithm proposed in [21], this method cannot be used when the user moves along the frontback direction. Furthermore, using the Graphics Processing 
Unit (GPU) implementation of this method, the calculation of a video hologram with $(1280 \times 768)$ pixels still took over $0.1 \mathrm{~s}$ per frame for a scene containing 9243 points, which is too slow for AR applications [22].

In this paper, we propose a real-time CGH calculation method for the FTOS, which can also be used for a spherically illuminated holographic display. Whereas previously proposed methods used a ray-tracing approach to compensate the distortion induced by the converging lens, our method relies on a layer-based approach. Several layer-based hologram synthesis methods can be found in the literature [23-25]. However, since they were all proposed for the conventional optical system illuminated by a plane wave, these methods cannot be used to compensate the distortion induced by a converging lens or a spherical illuminating light wave. Thanks to this approach, our method is able to compute $4 \mathrm{k} 2 \mathrm{k}$ holograms in real-time at 24 frames per second, paving the way for AR applications.

Section 2 gives a background on the FTOS, Section 3 and Section 4 present our proposed method and its implementation as a plugin for Unity, and experimental results are analyzed in Section 5 .

\section{Fourier transform optical sys- tem}

\section{$2.1 \quad$ Optical system}

The optical system used in conventional holographic HMDs $[13,14]$ is shown in Figure 1a. In these systems, the hologram is displayed on a beam shaping device called Spatial Light Modulator (SLM), which is illuminated by a coherent light beam. SLMs are electrically or optically addressed displays which reproduce the light wave scattered by a given scene by modulating the amplitude and/or the phase of the illuminating light beam.

Regardless of the technology they use, SLMs need small pixels to achieve their diffractive behavior and to provide a large FOV. Indeed, the maximum viewing angle provided by a SLM is given by

$$
\theta=\arcsin \left(\frac{\lambda}{2 p}\right)
$$

where $\lambda$ is the wavelength of the illuminating light beam and $p$ is the SLM pixel pitch. Unfortunately, current SLMs have pixel pitches of over 10 times the wavelength of visible light, which limit the FOV to a few degrees.

When using HMDs, the eyes of the viewer are located at a fixed distance from the SLM. In this case, the FOV limitation can be solved using the Fourier Transform Optical System (FTOS) [15]. The FTOS arrangement is shown in Figure 1b. In this display system, a converging lens of focal length $f$ is set at distance $\Delta$ in front of the SLM. The size of the SLM is given by

$$
\left(S_{x}, S_{y}\right)=\left(N_{x} p, N_{y} p\right),
$$

where $\left(N_{x}, N_{y}\right)$ is the SLM resolution.

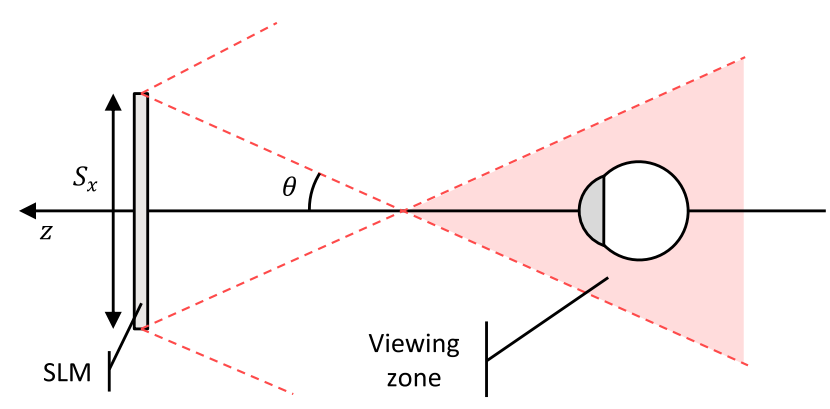

(a)

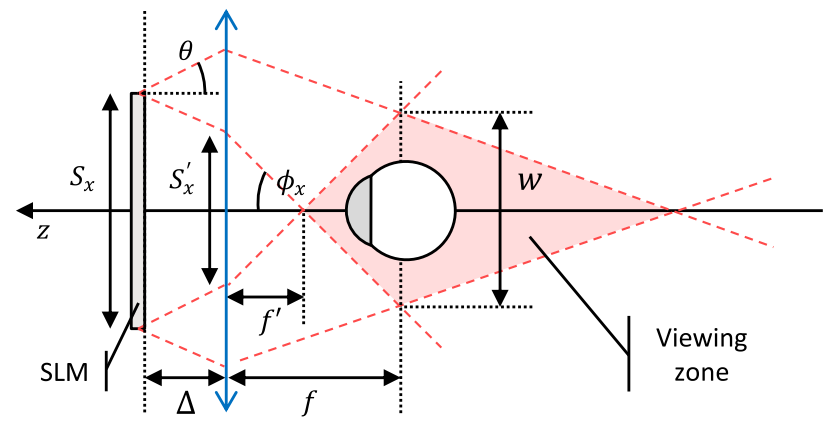

(b)

Figure 1: Holographic display systems: (a) conventional optical system, (b) Fourier Transform Optical System

The maximum FOV of this system is therefore given by

$$
\left\{\begin{array}{l}
\phi_{x}=\arctan \left(\frac{S_{x}^{\prime}}{2 f^{\prime}}\right) \\
\phi_{y}=\arctan \left(\frac{S_{y}^{\prime}}{2 f^{\prime}}\right) .
\end{array}\right.
$$

in the horizontal and vertical plane, respectively, with

$$
\begin{aligned}
S_{x}^{\prime} & =S_{x}-2 \Delta \tan (\theta), \\
S_{y}^{\prime} & =S_{y}-2 \Delta \tan (\theta), \\
f^{\prime} & = \begin{cases}\frac{S_{x}^{\prime}}{S_{x}^{\prime}+w} f, & \text { if } S_{x} \geq S_{y} \\
\frac{S_{y}^{\prime}}{S_{y}^{\prime}+w} f, & \text { otherwise },\end{cases} \\
w & =2 f \tan (\theta) .
\end{aligned}
$$

As shown in (3), the FOV can be enlarged by reducing the focal length of the lens or by increasing the SLM resolution. Moreover, it reaches its maximum value when $\Delta=0$. For instance, using a SLM of resolution $(1920 \times 1080)$ with a pixel pitch of $8 \mu \mathrm{m}$ and an illuminating light beam of $633 \mathrm{~nm}$, the FOV of the conventional optical system would be limited to $4.5^{\circ}$. Using the FTOS with a converging lens of focal length $6 \mathrm{~cm}$, the FOV is enlarged up to $19.0^{\circ}$ and $10.8^{\circ}$ in the horizontal and vertical plane, respectively.

In the following, we consider $\Delta=0$. We therefore have

$$
\left(S_{x}^{\prime}, S_{y}^{\prime}\right)=\left(S_{x}, S_{y}\right) .
$$




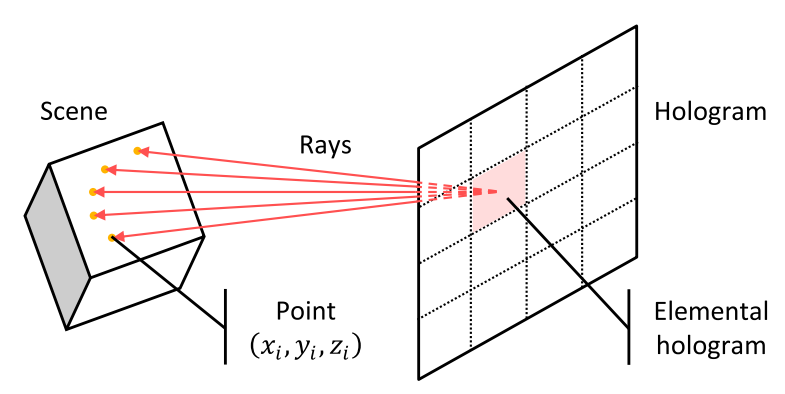

Figure 2: Conventional hologram calculation using the raytracing approach.

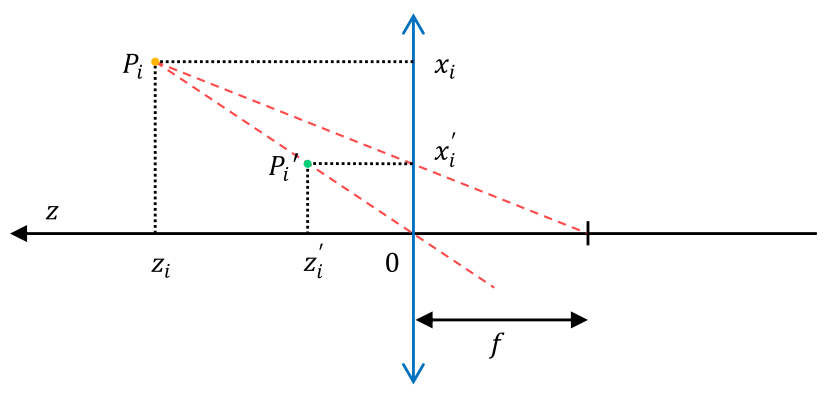

Figure 3: Compensation of distortion induced by the Fourier Transform Optical System.

It must be noted that the FTOS is equivalent to a conventional optical system illuminated by a spherical wave converging to a point located at distance $f$ from the SLM.

\subsection{Conventional calculation method}

As shown in the previous section, the FOV of holographic HMDs can be enlarged using the FTOS. However, since the linear magnification of virtual objects depends on their depth, the geometry of the scene reconstructed by the hologram is distorted by the converging lens. It is therefore necessary to compensate this distortion during the hologram calculation.

In $[21,26]$, the authors computed the hologram for the FTOS using a ray-tracing approach. In this method, the hologram is partitioned into several segments called elementary holograms, as shown in Figure 2. From the center of each elementary hologram, rays are cast into the scene volume, and their intersection with object surfaces produces a collection of points which are considered as spherical light sources. Then, the coordinates of these points are modified to compensate the distortion induced by the FTOS, as shown in Figure 3.

For each scene point $P_{i}$ located at $\left(x_{i}, y_{i}, z_{i}\right)$, its compensated coordinates are given by

$$
\left(\begin{array}{l}
x_{i}^{\prime} \\
y_{i}^{\prime} \\
z_{i}^{\prime}
\end{array}\right)=\left(\begin{array}{c}
\frac{f}{z_{i}+f} x_{i} \\
\frac{f}{z_{i}+f} y_{i} \\
\frac{f}{z_{i}+f} z_{i}
\end{array}\right) .
$$

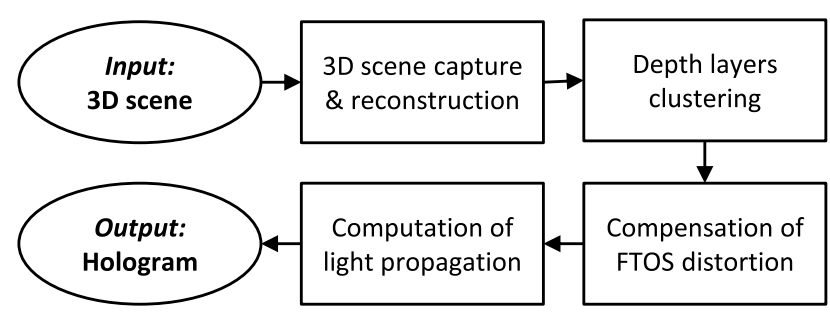

Figure 4: Overall block-diagram of the proposed method.

Finally, each elementary complex hologram $H_{e}$ is computed as the sum of spherical light waves scattered by the points, such that

$$
H_{e}(x, y)=\sum_{i=0}^{N_{e}-1} \frac{A_{i}}{r_{i}} \exp \left[j\left(\frac{2 \pi}{\lambda} r_{i}+\phi_{i}\right)\right],
$$

where $N_{e}$ is the number of scene points for elementary hologram $e, \lambda$ is the wavelength of light, $A_{i}$ is the amplitude of point $i$, calculated using shading formulas, and $\phi_{i} \in[0 ; 2 \pi$ [ is its initial phase, set to a random value between 0 (included) and $2 p i$ (excluded) to render a diffuse scene. Finally, $r_{i}$ is the oblique distance, given by

$$
r_{i}=\sqrt{\left(x-x_{i}^{\prime}\right)^{2}+\left(y-y_{i}^{\prime}\right)^{2}+z_{i}^{\prime 2}} .
$$

Since hidden surfaces are removed during the ray casting process, this method properly takes into account occlusions between scene objects. Moreover, Computer Graphics shading techniques, such as Phong shading [27], can be integrated during the hologram calculation to produce photo-realistic effects [21]. However, the main drawback of this method is its very high computational complexity, since it requires one calculation per scene point per hologram pixel. In [22], the authors proposed to reduce the computational load by taking into account temporal redundancies during viewpoint parallel shift and rotation. However, this method cannot be used when the user moves along the front-back direction. Furthermore, since this method relies on the ray-tracing approach, its computational complexity is still very high, and real-time calculation at 24 frames per second or more was not achievable [22].

\section{Proposed calculation method}

In order to tackle these issues, we propose a cluster-based algorithm exploiting the non-uniform geometry distribution in space, allowing to reduce the computation time. Figure 4 shows the overall block-diagram of our method, which consists of four steps. First, the 3D scene geometry is captured and reconstructed as a collection of depth layers parallel to the hologram plane. Then, to accelerate the CGH calculation, the depth layers are clustered into a set of $N_{z}$ groups, each one being associated with a reference layer at a given depth. Then, the position and size of each reference depth layer is modified to compensate the distortion induced by the FTOS. 
Finally, light scattered by the scene is numerically propagated towards the hologram plane and encoded into the final CGH.

\subsection{Scene capture and reconstruction}

The first step of our proposed method is to capture and reconstruct the $3 \mathrm{D}$ scene geometry as a set of parallel depth layers. We first synthesize the 2D-plus-depth perspective projection of the scene from the viewer's viewpoint. To this end, we use a virtual camera with a FOV of $\phi_{x}$ and $\phi_{y}$ in the horizontal and vertical planes, respectively, and a resolution of $\left(N_{x}, N_{y}\right)$. The projection matrix of this camera is therefore given by

$$
M=\left[\begin{array}{cccc}
f^{\prime} / p & 0 & n_{0} & 0 \\
0 & f^{\prime} / p & m_{0} & 0 \\
0 & 0 & 1 & 0
\end{array}\right]
$$

where

$$
\left\{\begin{array}{l}
n_{0}=N_{x} / 2 \\
m_{0}=N_{y} / 2 .
\end{array}\right.
$$

Then, the 3D scene geometry is reconstructed from the acquired 2D-plus-depth data. We call $I$ and $D$ the intensity and depth images captured by the camera. Since $D$ is encoded as an 8-bits gray level image, each pixel $[n, m]$ is projected back to a $3 \mathrm{D}$ point whose coordinates are given by

$$
\left(\begin{array}{l}
x_{n} \\
y_{m} \\
z_{d}
\end{array}\right)=\left(\begin{array}{c}
\frac{\left(z_{d}+f^{\prime}\right)\left(n-n_{0}\right) p}{f^{\prime}} \\
\frac{\left(z_{d}+f^{\prime}\right)\left(m-m_{0}\right) p}{f^{\prime}} \\
\frac{d}{255}\left(z_{\max }-z_{\min }\right)+z_{\min }
\end{array}\right)
$$

where $z_{\min }$ and $z_{\max }$ are the minimal and maximal depths recorded in $D$, and $d=D[n, m]$. The projected 3D points are thus naturally sliced into 256 evenly distributed depth layers.

\subsection{Depth layers clustering}

In the second step of our proposed method, the layers are clustered into a set of $N_{z} \leq 256$ groups defined by their depth intervals $\left[d_{i} ; d_{i+1}\left[\right.\right.$, with $i \in\left[0 ; N_{z}[\right.$. The objective of this clustering is to accelerate the $\mathrm{CGH}$ calculation by reducing the number of depth layers with a minimal decrease of visual quality. To this end, we use the k-means clustering algorithm, which is described in the following.

First, the histogram $\mathcal{H}_{D}$ of $D$ is computed and the depth intervals are initialized such that

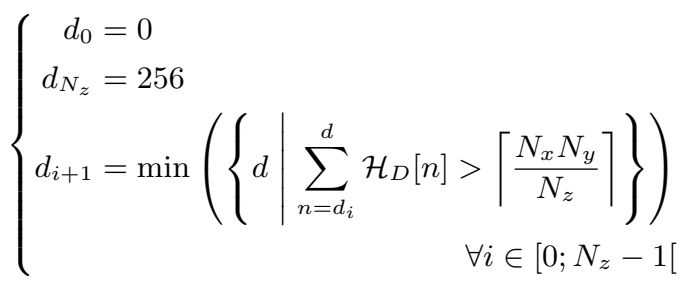

where $\lceil a\rceil$ is the least integer greater than or equal to $a$. For each cluster of layers $i \in\left[0 ; N_{z}\left[\right.\right.$, the average depth $\overline{d_{i}}$ is thus given by

$$
\overline{d_{i}}=\sum_{d=d_{i}}^{d_{i+1}-1} \frac{\mathcal{H}_{D}[d] d}{N_{i}}
$$

where

$$
N_{i}=\sum_{d=d_{i}}^{d_{i+1}-1} \mathcal{H}_{D}[d]
$$

is the total number of scene points within cluster $i$.

Then, the clusters are iteratively refined by alternating between two steps: an assignment step and an update step. During the assignment step, the depth intervals are modified such that

$$
d_{i+1}=\min \left(\left\{d|| d-\overline{d_{i}}|>| d-\overline{d_{i+1}} \mid\right\}\right) \forall i \in\left[0 ; N_{z}-1[.\right.
$$

During the update step, the mean depths $\overline{d_{i}}$ are updated according to (16). The assignment and update steps are repeated until the intervals no longer change.

Finally, each group of layers $i$ is associated with a single reference depth layer located at depth

$$
\overline{z_{i}}=\frac{\overline{d_{i}}}{255}\left(z_{\max }-z_{\min }\right)+z_{\min } .
$$

This reference depth layer is then considered to operate as a surface source of light which emits a complex wave $o_{i}$ sampled on a regular grid of resolution $\left(N_{x}, N_{y}\right)$, such that

$o_{i}[n, m]= \begin{cases}\sqrt{I[n, m]} \exp (j \phi[n, m]), & \text { if } D[n, m] \in\left[d_{i} ; d_{i+1}[\right. \\ 0, & \text { otherwise, }\end{cases}$

where $\phi[n, m] \in[0 ; 2 \pi[$ is the initial phase, set to a uniform random value to render a diffuse scene.

\subsection{Compensation of FTOS distortion}

The third step of our proposed method is to compensate the distortion induced by the FTOS by modifying the position and size of each reference layer. The geometrical relation between the compensated layers used during the hologram computation and the perceived reference layers magnified by the FTOS is given in Figure 5.

According to (9), the depth of compensated layer $i$ is given by

$$
{\overline{z_{i}}}^{\prime}=\frac{f}{\overline{z_{i}}+f} \overline{z_{i}} .
$$

Furthermore, we call

$$
\gamma_{i}=\frac{p}{p_{i}}=\frac{f^{\prime \prime}}{f^{\prime \prime}+{\overline{z_{i}}}^{\prime}}=\frac{f^{\prime}\left(\overline{z_{i}}+f\right)}{f\left(\overline{z_{i}}+f^{\prime}\right)}
$$

the linear magnification between the hologram and the compensated layer $i$, where

$$
f^{\prime \prime}=\frac{f f^{\prime}}{f-f^{\prime}} .
$$

Substituting (6) and (8) into (23), $f^{\prime \prime}$ becomes

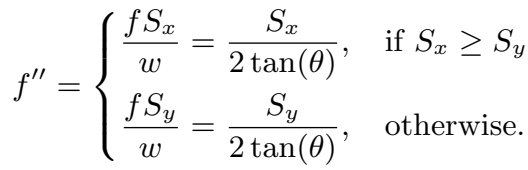




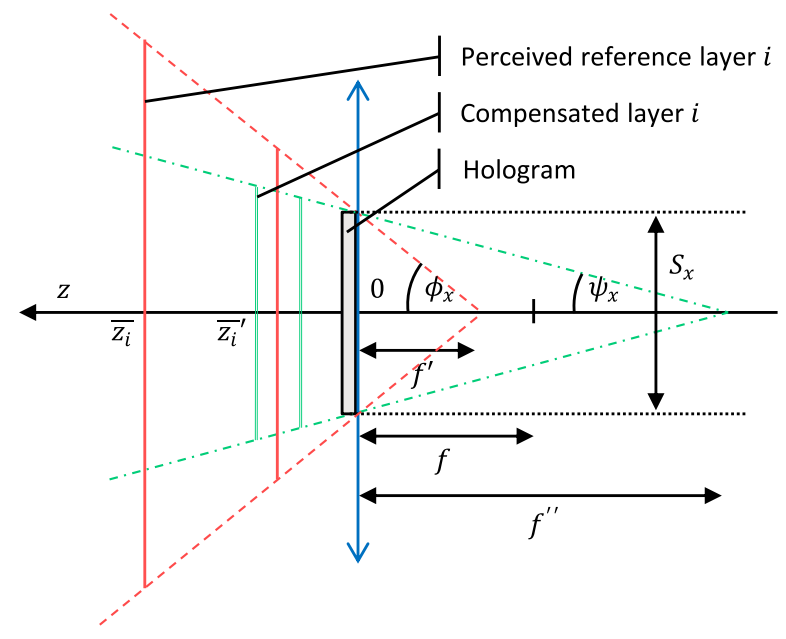

Figure 5: Geometrical relation between the compensated layers used during the hologram computation (in green) and the perceived reference layers magnified by the FTOS (in red).

The sampling pitch $p_{i}$ on compensated layer $i$ is therefore given by

$$
\begin{aligned}
& p_{i}=\frac{p}{\gamma_{i}} \\
& p_{i}= \begin{cases}\frac{2\left(f^{\prime \prime}+{\overline{z_{i}}}^{\prime}\right) \tan \left(\arcsin \left(\frac{\lambda}{2 p}\right)\right)}{N_{x}}, & \text { if } S_{x} \geq S_{y} \\
\frac{2\left(f^{\prime \prime}+{\overline{z_{i}}}^{\prime}\right) \tan \left(\arcsin \left(\frac{\lambda}{2 p}\right)\right)}{N_{y}}, & \text { otherwise. }\end{cases}
\end{aligned}
$$

(24) and (26) can be simplified under the paraxial approximation, which is valid for $\lambda<0.5 p$. Indeed, under this assumption, we have

$$
\tan \left(\arcsin \left(\frac{\lambda}{2 p}\right)\right) \approx \frac{\lambda}{2 p}
$$

with an error under 3.5\%. Substituting (27) into (24) and (26), we get

$$
f^{\prime \prime} \approx \begin{cases}\frac{N_{x} p^{2}}{\lambda}, & \text { if } S_{x} \geq S_{y} \\ \frac{N_{y} p^{2}}{\lambda}, & \text { otherwise }\end{cases}
$$

and

$$
p_{i} \approx \begin{cases}\frac{\left(f^{\prime \prime}+{\overline{z_{i}}}^{\prime}\right) \lambda}{N_{x} p}, & \text { if } S_{x} \geq S_{y} \\ \frac{\left(f^{\prime \prime}+{\overline{z_{i}}}^{\prime}\right) \lambda}{N_{y} p}, & \text { otherwise. }\end{cases}
$$

\subsection{Computation of light propagation}

In the last step of our method, the light waves scattered by the compensated depth layers are numerically propagated towards the hologram plane and summed up to get the CGH

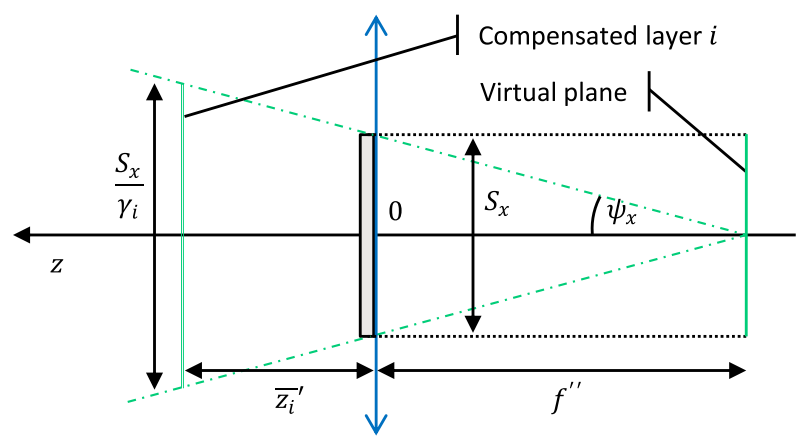

Figure 6: Computation of the hologram: the light waves scattered by the compensated depth layers are propagated towards a unique virtual plane. They are then summed up and propagated to the hologram plane.

$H$, such that

$$
H[n, m]=\sum_{i=0}^{N_{z}-1} \mathcal{P}_{{\overline{z_{i}}}^{\prime}, \gamma_{i}}\left\{o_{i}\right\}[n, m],
$$

where operator $\mathcal{P}_{z, \gamma}$ stands for the numerical propagation of light between two parallel planes separated by a distance $z$ with sampling pitch magnification $\gamma$.

Several algorithms have been proposed to allow this magnification to be adjusted during propagation. One of the most commonly used is the Double-Step Fresnel diffraction (DSF) method [28], given by

$$
\mathcal{P}_{{\overline{z_{i}}}^{\prime}, \gamma_{i}}\left\{o_{i}\right\}=S S F_{z_{i, 2}}\left\{S S F_{z_{i, 1}}\left\{o_{i}\right\}\right\}
$$

where $z_{i, 1}$ and $z_{i, 2}$ are intermediate reconstruction distances, defined in the following, and $S S F_{z}$ stands for the Fresnel diffraction, given by

$S S F_{z}\left\{o_{i}\right\}(x, y)=\frac{e^{j \frac{2 \pi}{\lambda} z}}{j \lambda z} e^{j \frac{\pi}{\lambda z}\left(x^{2}+y^{2}\right)} \mathcal{F}^{\operatorname{sgn}(z)}\left\{o_{i}(\xi, \eta) e^{j \frac{\pi}{\lambda z}\left(\xi^{2}+\eta^{2}\right)}\right\}$

In $(32), \mathcal{F}^{\mathrm{sgn}(z)}$ is the forward or inverse Fourier Transform, when $z$ is positive or negative, respectively. This transform can be computed using the Fast Fourier Transform (FFT) algorithm [29]. In the Fresnel diffraction, the sampling pitches in the destination plane are given by

$$
\left(p_{x}^{\prime}, p_{y}^{\prime}\right)=\left(\frac{\lambda|z|}{N_{x} p_{x}}, \frac{\lambda|z|}{N_{y} p_{y}}\right),
$$

where $\left(p_{x}, p_{y}\right)$ are the sampling pitches in the source plane and $z$ is the propagation distance. As a consequence, using the DSF, the sampling pitch magnification $\gamma_{i}$ can be adjusted by setting the intermediate reconstruction distances such that

$$
\left\{\begin{array}{l}
z_{i, 1}=\frac{{\overline{z_{i}}}^{\prime}}{\gamma_{i}+1} \\
z_{i, 2}=\frac{\gamma_{i}{\overline{z_{i}}}^{\prime}}{\gamma_{i}+1} .
\end{array}\right.
$$




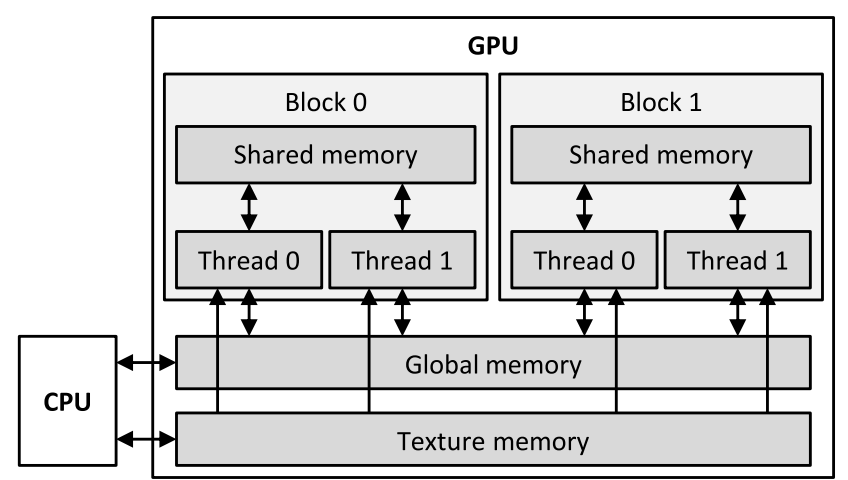

Figure 7: CUDA thread organization and memory model

Thanks to the FFT algorithm, the DSF propagation is computationally efficient. Nevertheless, to accelerate the CGH calculation further, we set the intermediate reconstruction distances to

$$
\left\{\begin{array}{l}
z_{i, 1}={\overline{z_{i}}}^{\prime}+f^{\prime \prime} \\
z_{i, 2}=-f^{\prime \prime}
\end{array}\right.
$$

According to (33), the sampling pitches in the hologram plane are therefore given by

$$
\left\{\begin{array}{l}
p_{x}^{\prime \prime}=\frac{\lambda f^{\prime \prime}}{N_{x} \frac{\lambda\left(f^{\prime \prime}+\bar{z}_{i}\right.}{N_{x} p_{i}}}=\frac{f^{\prime \prime} p_{i}}{f^{\prime \prime}+\overline{z_{i}}} \\
p_{y}^{\prime \prime}=\frac{\lambda f^{\prime \prime}}{N_{y} \frac{\lambda\left(f^{\prime \prime}+\bar{z}_{i}^{\prime}\right)}{N_{y} p_{i}}}=\frac{f^{\prime \prime} p_{i}}{f^{\prime \prime}+\overline{z_{i}}} .
\end{array}\right.
$$

By substituting (28) and (29) in (36), we get

$$
p_{x}^{\prime \prime}=p_{y}^{\prime \prime} \approx p \text {. }
$$

We thus verify that the sampling pitch in the hologram plane corresponds to the pixel pitch of the CGH.

Using these intermediate reconstruction distances, the light waves scattered by the compensated depth layers are therefore propagated towards a unique virtual plane located at distance $f^{\prime \prime}$ from the hologram, as shown in Figure 6. They are then summed up and propagated to the hologram plane to get the CGH $H$, such that

$$
H[n, m]=S S F_{-f^{\prime \prime}}\left\{\sum_{i=0}^{N_{z}-1} S S F_{\bar{z}^{\prime}}+f^{\prime \prime}\left\{o_{i}\right\}\right\}[n, m] .
$$

We notice that using (38), we need only to compute one FFT per compensated depth layer, whereas two were needed using (31). The computational complexity of (38) is therefore divided by two compared to (30), when $\mathcal{P}_{z, \gamma}$ is calculated using (31).

\section{Graphics Processing Unit im- plementation}

The proposed method was implemented using the Unity game engine on a PC system employing an operating system of Mi-

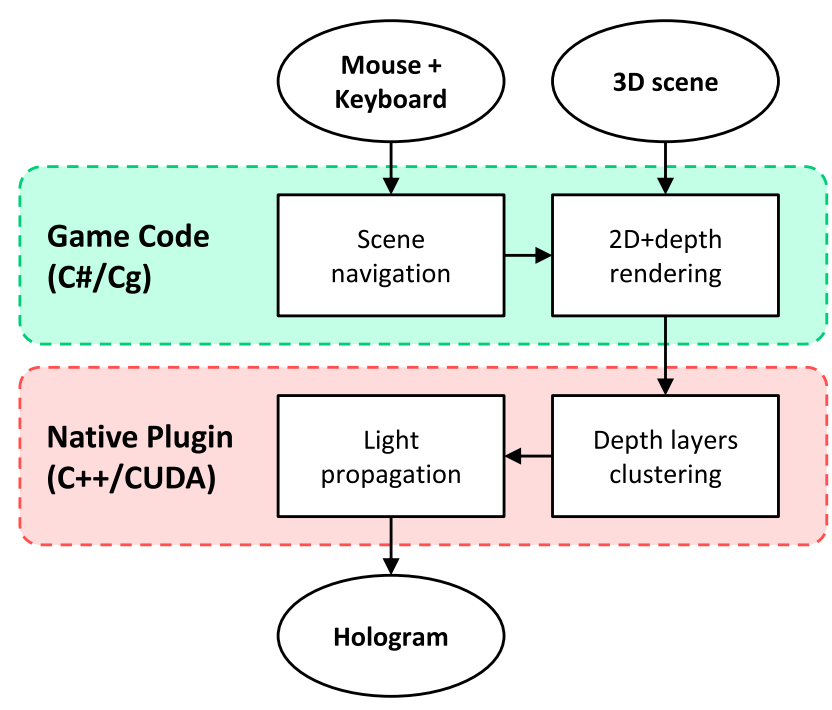

Figure 8: Block diagram of our proposed implementation.

crosoft Windows 8, an Intel Core i7-4930K CPU operating at $3.40 \mathrm{GHz}$, a main memory of $16 \mathrm{~GB}$ and a GPU NVIDIA GeForce GTX 780Ti. In our implementation, all the CGH computation is performed by the GPU using the CUDA application programming interface.

\subsection{CUDA thread organization and memory model}

Figure 7 shows the CUDA thread organization and memory model used by the GPU. The parallel code portions which are executed on the GPU are called kernels. Each kernel is composed by several threads, which are organized in blocks. Threads within a single block can exchange data through the on-chip shared memory, which has a short-latency but limited capacity. The CPU and GPU threads exchange data through the global memory, which has a large capacity but long latency and limited bandwidth. Additionally, the GPU threads can also access to a read-only texture memory, which is cached on-chip. The texture cache is optimized for $2 \mathrm{D}$ spatial locality, so threads that read texture addresses that are close together will achieve best performance.

\subsection{Implementation as a native plugin for Unity}

Figure 8 shows the overall block diagram of our implementation, which consists of two parts: the Unity engine game code, written in $\mathrm{C \#}$ and $\mathrm{Cg}$, and the native plugin code, written in $\mathrm{C}++$ and CUDA.

The game code manages the user's keyboard and mouse navigation. A script, written in C\#, updates the user's position and orientation according to the keyboard and mouse inputs. Then, once per frame, the $2 \mathrm{D}$-plus-depth perspective projection of the scene is rendered from the user's viewpoint 


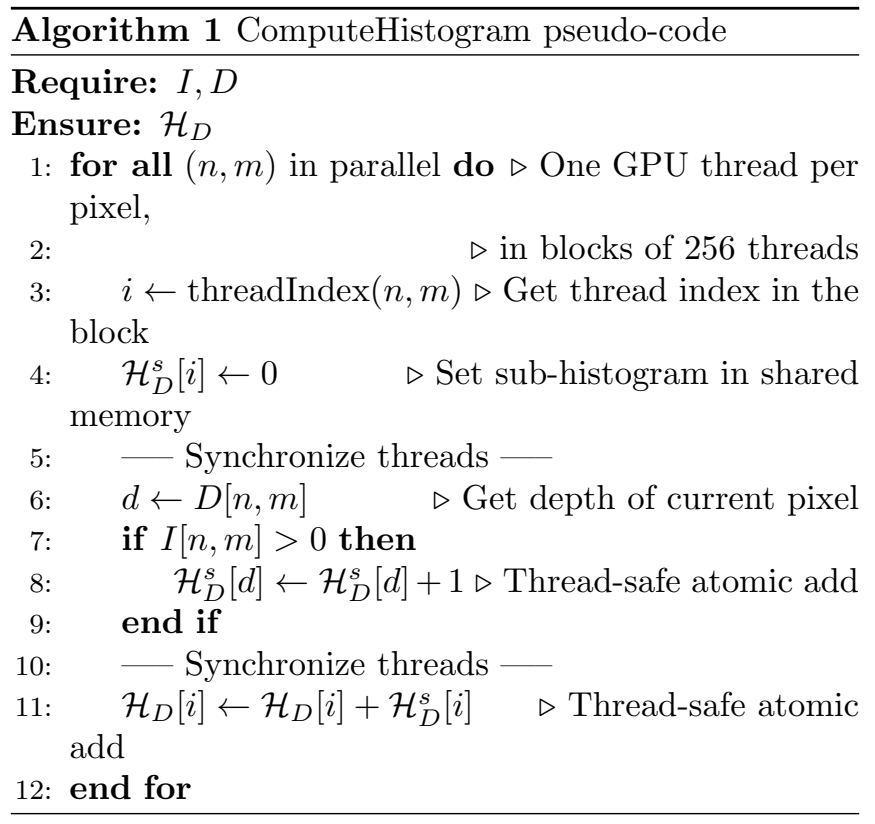

\begin{tabular}{|l|l|}
\hline \multicolumn{1}{|c|}{ Parameter } & \multicolumn{1}{c|}{ Value } \\
\hline Resolution $\left(N_{x} \times N_{y}\right)$ & $(3840 \times 2160)$ \\
\hline Pixel pitch $(p)$ & $3.74 \mu \mathrm{m}$ \\
\hline Light wavelength $(\lambda)$ & $632.8 \mathrm{~nm}$ \\
\hline FTOS focal length $(f)$ & $6 \mathrm{~cm}$ \\
\hline Horizontal field of view $\left(2 \phi_{x}\right)$ & $23.1^{\circ}$ \\
\hline Vertical field of view $\left(2 \phi_{y}\right)$ & $13.1^{\circ}$ \\
\hline Viewing zone size $(w)$ & $1 \mathrm{~cm}$ \\
\hline
\end{tabular}

Table 1: Optical system parameters used for the experiments.

using a virtual camera with a FOV of $\phi_{x}$ and $\phi_{y}$ in the horizontal and vertical planes, respectively, and a resolution of $\left(N_{x}, N_{y}\right)$. Finally, the game code calls the native plugin to compute the hologram from the acquired 2D-plus-depth projection.

First of all, the depth map histogram $\mathcal{H}_{D}$ is computed on the GPU by kernel ComputeHistogram, whose pseudo-code is given in Algorithm 1. ComputeHistogram is executed in parallel using one GPU thread per pixel, organized in blocks of 256 threads. Each parallel block computes a separate subhistogram $\mathcal{H}_{D}^{s}$ stored in shared memory. The threads are then synchronized and the resulting sub-histograms are summed up to obtain the global depth histogram $\mathcal{H}_{D}$. To avoid race conditions, the increment and summation operations are performed using atomic operations. The depth histogram is then transferred to the CPU memory and the depth layers are clustered and combined into a set of $N_{z} \leq 256$ compensated depth layers.

Finally, the light waves scattered by these compensated depth layers are numerically propagated to the hologram plane to obtain the $\mathrm{CGH} H$. To this end, the inner products in (32) are computed on the GPU using one CUDA thread per sample and the Fourier transforms are calculated using

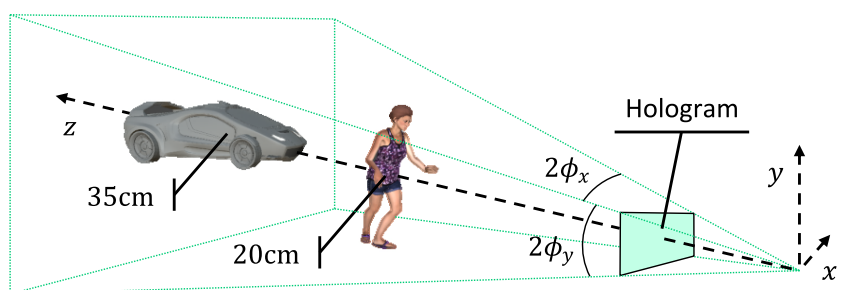

Figure 9: Overview of the Dancer scene layout.

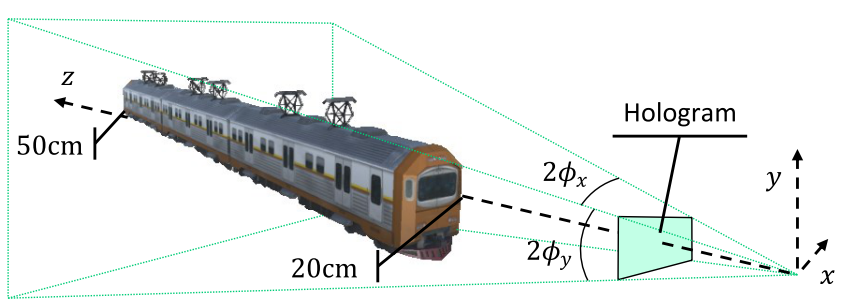

Figure 10: Overview of the Train scene layout.

the CUDA cuFFT library by NVIDIA. This library uses the Cooley-Tukey algorithm [29] to optimize the performance of any transform size that can be factored as $2^{a} 3^{b} 5^{c} 7^{d}$, where $a$, $b, c$ and $d$ are non-negative integers.

\section{Experimental results}

Table 1 shows the optical system parameters used for the experiments. The computed hologram is displayed on a SLM from Jasper Display Corp. with a resolution of $(3840 \times 2160)$, a pixel pitch of $3.74 \mu \mathrm{m}$, and is illuminated by a laser source with wavelength $632.8 \mathrm{~nm}$. For the FTOS we used a converging lens with a focal length of $6 \mathrm{~cm}$, enlarging the field of view up to $23.1^{\circ}$ and $13.1^{\circ}$ in the horizontal and vertical plane, respectively, for a viewing zone size of $1 \mathrm{~cm}$.

\subsection{Optical reconstructions}

To evaluate the optical reconstructions quality, we used two different 3D scenes: Dancer and Train, whose layouts are shown in Figures 9 and 10, respectively. Dancer is used to assess the performance of our method on scenes containing several isolated objects. It contains two 3D models: an animated dancing character and a car, located at $20 \mathrm{~cm}$ and $35 \mathrm{~cm}$ from the viewer, respectively. By contrast, Train is used to evaluate the limitations of our method on scenes containing large 3D objects spanning on a wide range of depth levels. It contains a single 3D train model located between $20 \mathrm{~cm}$ and $50 \mathrm{~cm}$ away from the viewer. To evaluate the optical reconstructions quality, we used four depth clusters. Figures 11 and 12 show the rendered intensity views and depth clusters arrangements of scenes Dancer and Train, respectively.

Figure 13 shows the optical reconstructions of the Dancer hologram recorded with a camera focusing on a varying depth. When the camera is focused on the dancing character, the car 


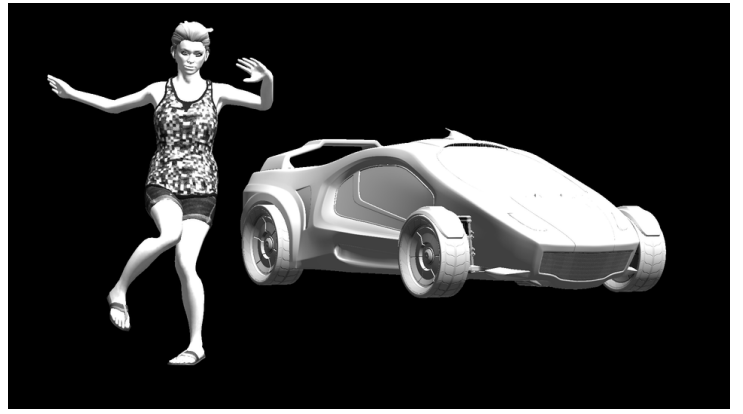

(a)

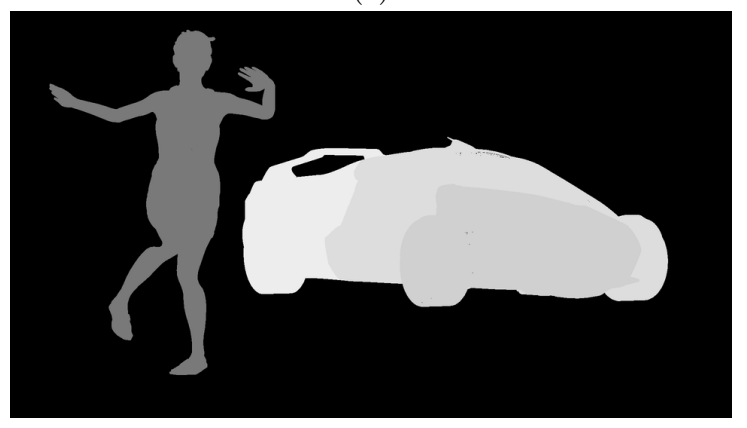

(b)

Figure 11: Input 2D+depth data of scene Dancer: (a) rendered intensity view, (b) depth clusters.

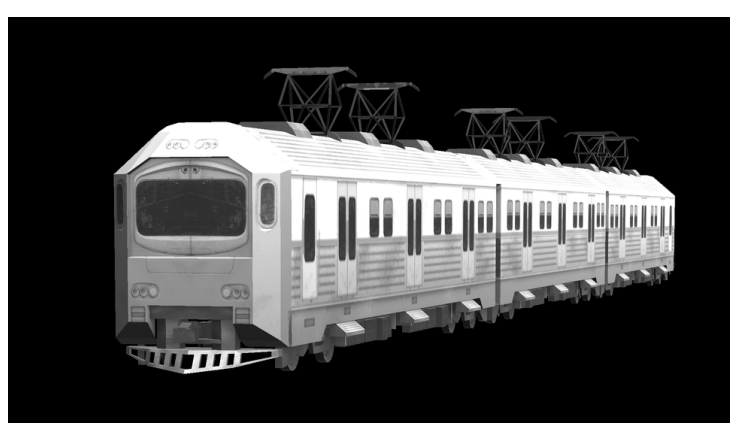

(a)

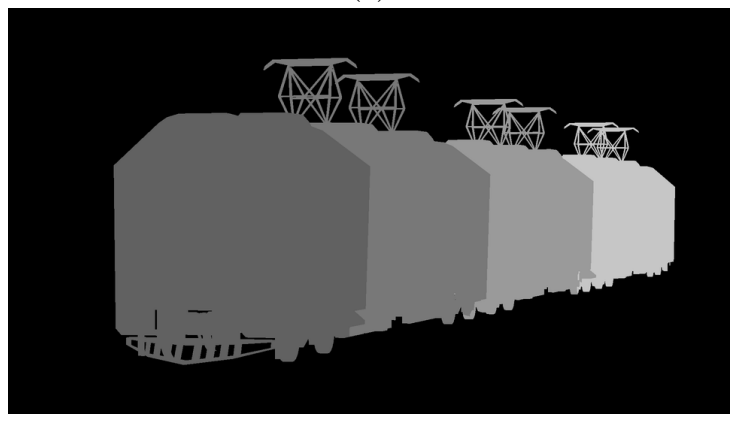

(b)

Figure 12: Input 2D+depth data of scene Train: (a) rendered intensity view, (b) depth clusters.

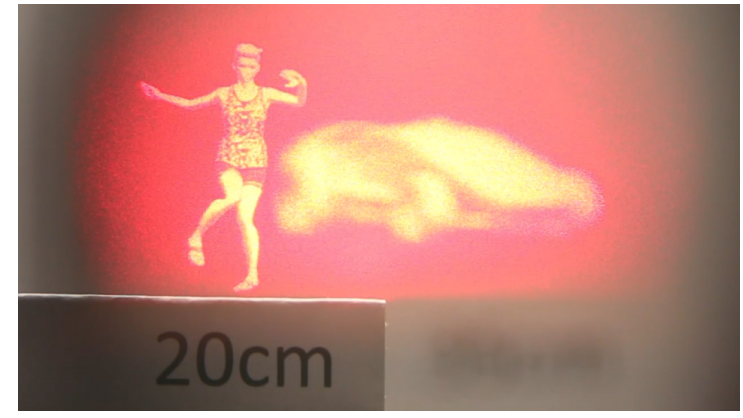

(a)

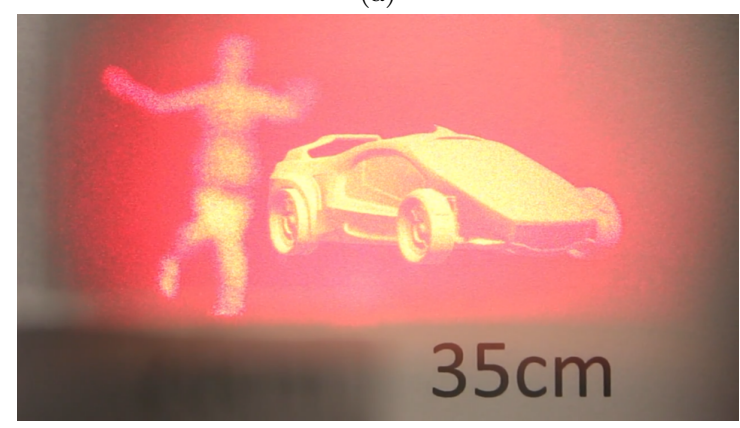

(b)

Figure 13: Optical reconstructions of scene Dancer: (a) focused on the dancer, (b) focused on the car.

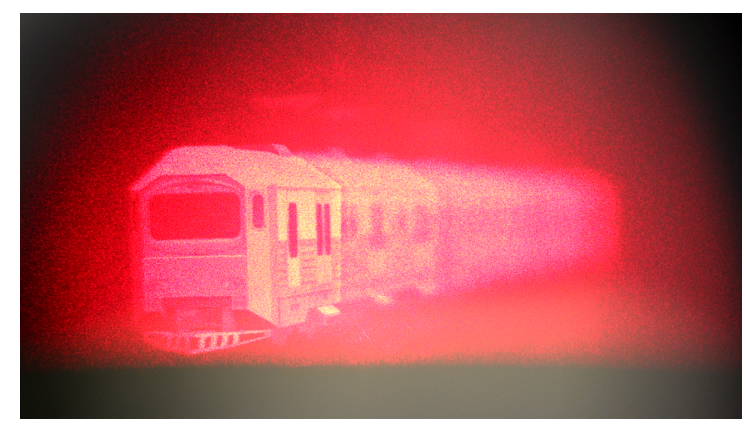

(a)

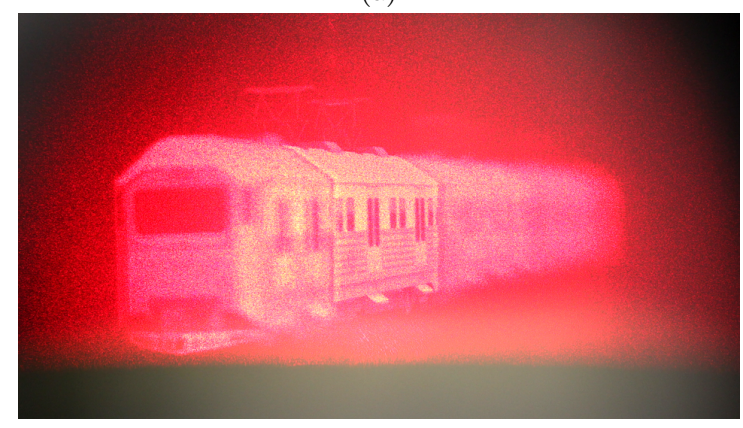

(b)

Figure 14: Optical reconstructions of scene Train: (a) focused on the 1st cluster, (b) focused on the 2 nd cluster. 
appears blurred, and vice versa. This demonstrates that our proposed method provides an accurate accommodation cue. Furthermore, thanks to the compensation step, the scene geometry is not distorted by the FTOS. Finally, the separation between the consecutive depth clusters composing the car is not noticeable, providing an almost continuous depth change.

The optical reconstructions of the Train hologram are shown in Figure 14. Since the 3D train model covers a wide range of depth levels, the separation between consecutive depth clusters is visible, showing the limitations of the depth clustering algorithm for continuous elongated objects. To solve this limitation, the number of depths clusters can be increased, but in this case the calculation time would also be increased, as shown in the following section.

\subsection{Analysis of the calculation time}

To evaluate the computation performance of our method, we measured the average hologram calculation time per frame for two different resolutions and different numbers of depth clusters. The measured calculation times of holograms Dancer and Train are shown in Figure 15.

As shown in this figure, the hologram calculation time is almost the same for both scenes and only varies with the resolution and number of depth clusters. This means that the calculation time of our method does not depend on the scene geometry and layout. Furthermore, it increases linearly with the number of depth clusters.

For a hologram of resolution $(3840 \times 2160)$ and when the number of depth clusters is less than 7 , the calculation time remains below 41ms, which corresponds to a framerate of 24fps. Similarly, for a hologram of resolution $(1920 \times 1080)$ and when the number of depth clusters is less than 32 , the calculation time per frame remains below $40 \mathrm{~ms}$, corresponding to a framerate of $25 \mathrm{fps}$. Moreover, by reducing the number of depth clusters, it is possible to improve the hologram calculation time. For example, for 12 depth clusters, the full-HD hologram calculation framerate increases up to $63 \mathrm{fps}$, and for 5 depth clusters, it reaches $120 \mathrm{fps}$. This enables the use of our method in Augmented Reality applications.

In addition to these experiments, we measured the calculation time of each step of our method for both 3D scenes. The measurements showed that the computation times of the $3 \mathrm{D}$ scene rendering and depth clustering steps took approximately $10 \%$ of the total hologram calculation time, regardless of the resolution and number of depth clusters. The remaining $90 \%$ correspond to the propagation of light scattered by the compensated depth layers.

\section{Conclusion}

In this paper we proposed a fast hologram calculation method for the FTOS built on a layer-based approach. Our method consists of four steps. First, the scene geometry is captured and reconstructed as a collection of depth layers parallel to the hologram plane. Then, the depth layers are grouped into depth clusters, each cluster being associated with a reference

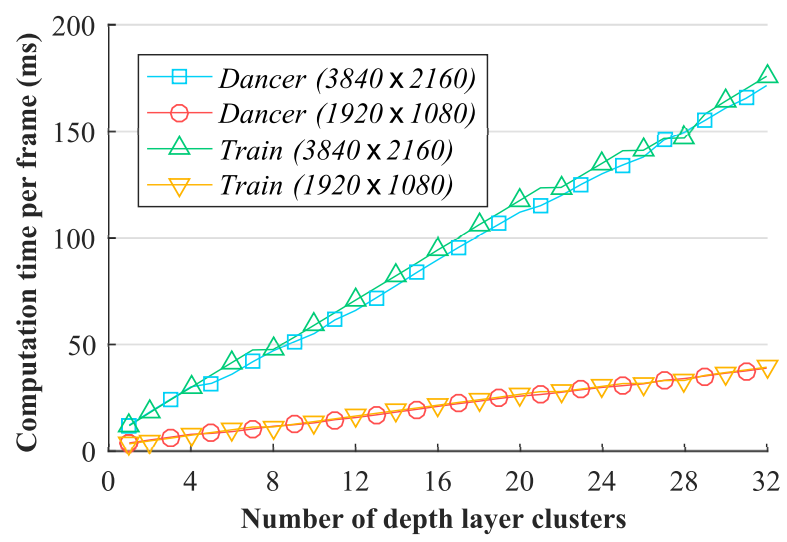

Figure 15: Hologram calculation time per frame.

layer at a given depth. Then, the position and size of each reference depth layer is modified to compensate the distortion induced by the FTOS. Finally, light scattered by the scene is numerically propagated towards the hologram plane and encoded into the final hologram.

Experimental results reveal that our method is able to compute $4 \mathrm{k} 2 \mathrm{k}$ holograms in real-time at 24 frames per second and full-HD holograms at more than 60 frames per second when the number of depth clusters is less than 7 and 12, respectively. This paves the way for Augmented Reality applications.

\section{Acknowledgment}

This work has been achieved within the Institute of Research and Technology $b<>$ com, dedicated to digital technologies. It has been funded by the French government through the National Research Agency (ANR) Investment referenced ANRA0-AIRT-07.

The Stefani 3D model and dancing animation are courtesy of Adobe Systems Inc. (www.mixamo.com). The car 3D model is courtesy of Unity Technologies (unity3d.com). The train 3D model is courtesy of TurboSquid (www.turbosquid.com).

\section{References}

[1] Julie Carmigniani, Borko Furht, Marco Anisetti, Paolo Ceravolo, Ernesto Damiani, and Misa Ivkovic. Augmented reality technologies, systems and applications. Multimedia Tools and Applications, 51(1):341-377, January 2011.

[2] Rong Wen, Wei-Liang Tay, Binh P. Nguyen, Chin-Boon Chng, and Chee-Kong Chui. Hand Gesture Guided Robot-assisted Surgery Based on a Direct Augmented Reality Interface. Comput. Methods Prog. Biomed., 116(2):68-80, September 2014. 
[3] A. H. Behzadan and V. R. Kamat. Visualization of construction graphics in outdoor augmented reality. In Proceedings of the Winter Simulation Conference, 2005., pages 7 pp.-, December 2005.

[4] Wolfgang Narzt, Gustav Pomberger, Alois Ferscha, Dieter Kolb, Reiner Müller, Jan Wieghardt, Horst Hörtner, and Christopher Lindinger. Augmented reality navigation systems. Universal Access in the Information Society, 4:177-187, March 2006.

[5] Mark A. Livingston, Lawrence J. Rosenblum, Simon J. Julier, Dennis Brown, Yohan Baillot, J. E. Swan, Joseph L. Gabbard, and Deborah Hix. An augmented reality system for military operations in urban terrain. In Proceedings of the Interservice / Industry Training, Simulation, \& Education Conference (I/ITSEC '02), page 89, Orlando, Florida, December 2002.

[6] David M. Hoffman, Ahna R. Girshick, Kurt Akeley, and Martin S. Banks. Vergence-accommodation conflicts hinder visual performance and cause visual fatigue. Journal of Vision, 8(3):33, March 2008.

[7] D. Dunn, C. Tippets, K. Torell, P. Kellnhofer, K. Akşit, P. Didyk, K. Myszkowski, D. Luebke, and H. Fuchs. Wide Field Of View Varifocal Near-Eye Display Using See-Through Deformable Membrane Mirrors. IEEE Transactions on Visualization and Computer Graphics, 23(4):1322-1331, April 2017.

[8] W. Wu, P. Llull, I. Tosic, N. Bedard, K. Berkner, and N. Balram. Content-adaptive focus configuration for near-eye multi-focal displays. In 2016 IEEE International Conference on Multimedia and Expo (ICME), pages 1-6, July 2016.

[9] Nathan Matsuda, Alexander Fix, and Douglas Lanman. Focal Surface Displays. ACM Trans. Graph., 36(4):86:186:14, July 2017.

[10] Hong Hua and Bahram Javidi. A 3d integral imaging optical see-through head-mounted display. Optics Express, 22(11):13484-13491, June 2014.

[11] Hyun-Eui Kim, Nam Kim, Hoon Song, Hong-Seok Lee, and Jae-Hyeung Park. Three-dimensional holographic display using active shutter for head mounted display application. pages $78631 \mathrm{Y}-78631 \mathrm{Y}-8$. International Society for Optics and Photonics, February 2011.

[12] Ulf Schnars and Werner Jüptner. Digital Holography: Digital Hologram Recording, Numerical Reconstruction, and Related Techniques. Springer Science \& Business Media, December 2005.

[13] Eunkyong Moon, Myeongjae Kim, Jinyoung Roh, Hwi Kim, and Joonku Hahn. Holographic head-mounted display with RGB light emitting diode light source. Optics Express, 22(6):6526-6534, March 2014.
[14] Qiankun Gao, Juan Liu, Xinhui Duan, Tao Zhao, Xin Li, and Peilin Liu. Compact see-through 3d head-mounted display based on wavefront modulation with holographic grating filter. Optics Express, 25(7):8412-8424, April 2017.

[15] Yuji Sakamoto. Portable three-dimensional display system for CGHs. volume 7233, page 723303. International Society for Optics and Photonics, February 2009.

[16] Liang Shi, Fu-Chung Huang, Ward Lopes, Wojciech Matusik, and David Luebke. Near-eye Light Field Holographic Rendering with Spherical Waves for Wide Field of View Interactive 3d Computer Graphics. ACM Trans. Graph., 36(6):236:1-236:17, November 2017.

[17] Tomasz Kozacki, Małgorzata Kujawińska, Grzegorz Finke, Bryan Hennelly, and Nitesh Pandey. Extended viewing angle holographic display system with tilted SLMs in a circular configuration. Applied Optics, 51(11):1771-1780, April 2012.

[18] Norbert Leister, Armin Schwerdtner, Gerald Fütterer, Steffen Buschbeck, Jean-Christophe Olaya, and Stanislas Flon. Full-color interactive holographic projection system for large 3d scene reconstruction. In Emerging Liquid Crystal Technologies III, volume 6911, page 69110V. International Society for Optics and Photonics, January 2008 .

[19] R. H. Y. Chen and T. D. Wilkinson. Field of view expansion for 3-D holographic display using a single spatial light modulator with scanning reconstruction light. In 2009 3DTV Conference: The True Vision - Capture, Transmission and Display of 3D Video, pages 1-4, May 2009 .

[20] Takuo Yoneyama, Eishin Murakami, Yuki Oguro, Hibiki Kubo, Kazuhiro Yamaguchi, and Yuji Sakamoto. Holographic head-mounted display with correct accommodation and vergence stimuli. Optical Engineering, 57(6):061619, May 2018.

[21] Tsubasa Ichikawa, Takuo Yoneyama, and Yuji Sakamoto. CGH calculation with the ray tracing method for the Fourier transform optical system. Optics Express, 21(26):32019-32031, December 2013.

[22] Ryosuke Watanabe, Kazuhiro Yamaguchi, and Yuji Sakamoto. Fast calculation method of computer generated hologram animation for viewpoint parallel shift and rotation using Fourier transform optical system. Applied Optics, 55(3):A167, January 2016.

[23] Yan Zhao, Liangcai Cao, Hao Zhang, Dezhao Kong, and Guofan Jin. Accurate calculation of computergenerated holograms using angular-spectrum layeroriented method. Optics Express, 23(20):25440, October 2015 . 
[24] Antonin Gilles, Patrick Gioia, Rémi Cozot, and Luce Morin. Hybrid approach for fast occlusion processing in computer-generated hologram calculation. Applied $\mathrm{Op}$ tics, 55(20):5459-5470, July 2016.

[25] Antonin Gilles, Patrick Gioia, Rémi Cozot, and Luce Morin. Computer generated hologram from Multiviewplus-Depth data considering specular reflections. In 2016 IEEE International Conference on Multimedia Expo Workshops (ICMEW), pages 1-6, July 2016.

[26] Tsubasa Ichikawa, Takuo Yoneyama, and Yuji Sakamoto. Full color realistic 3d image reconstruction in CGH with binocular vision. Proceedings of 3DSA2013, 6:3, 2013.

[27] Bui Tuong Phong. Illumination for Computer Generated Pictures. Communications of the ACM, 18(6):311-317, June 1975 .

[28] Fucai Zhang, Ichirou Yamaguchi, and L. P. Yaroslavsky. Algorithm for reconstruction of digital holograms with adjustable magnification. Optics Letters, 29(14):16681670, July 2004.

[29] James W. Cooley and John W. Tukey. An algorithm for the machine calculation of complex Fourier series. Mathematics of Computation, 19(90):297-301, 1965. 